\title{
ON LINEAR ORDINARY DIFFERENTIAL EQUATIONS WITH EXPONENTIAL COEFFICIENTS*
}

\author{
BY \\ G. A. HEGEMIER \\ University of California, San Diego
}

\begin{abstract}
A system of linear ordinary differential equations with exponential coefficients is considered; several theorems are presented concerning general solutions and their asymptotic forms for large values of the independent variable; elementary examples are given and applications cited to illustrate, and demonstrate the utility of, the solution forms obtained. The relevance of the results to the solution of problems of structural stability and wave propagation is briefly discussed.

1. Introduction. Differential equations with exponential coefficients occur frequently in the study of certain boundary and initial value problems in physics and engineering; consequently their solutions are of interest to the analyst. In this paper several theorems are presented concerning general solutions of such equations and their asymptotic behavior for large values of the independent variable.

The construction method employed here parallels that used by Valeev in reference [1] and the author in reference [3]. Accordingly, the problem of constructing a solution of the differential equations is reduced to a study of a system of linear functional difference equations by application of the Laplace transform to the former. It is shown that the operator associated with said difference equations forms a contraction mapping in a complete metric space. Applying a well-known theorem due to S. Banach on fixed points, the unique fixed point of the operator is generated by successive approximations. The desired solution of the differential equations is then obtained by a direct inversion of the transform. In the sequel, two forms of the inversion are given.

In Sec. 2 of this paper we present the differential equations to be studied. The main theorems are given in Sec. 3. In Sec. 4, we prove the main theorems and finally, in Sec. 5, we conclude with several illustrative examples. Applications of the results contained herein to the solution of a number of practical engineering problems are discussed in the closing remarks (see Sec. 6).
\end{abstract}

2. Differential equations. Let the system of differential equations

$$
\sum_{j=0}^{l} \sum_{k=0}^{n} \exp \left(-\alpha_{j} x\right) C_{j k} \frac{d^{k} Y(x)}{d x^{k}}=P(x)
$$

be given on the domain (of the real variable $x$ ) $x>0$, where $P(x)$ has the form

$$
P(x)=\sum_{\gamma=1}^{\lambda} P_{\gamma} \exp \left(\beta_{\gamma} x\right) \text {. }
$$

Here $l, n, \lambda$ are finite; $Y(x), P(x)$ are $m$-dimensional vectors; $P_{\gamma}$ are complex-valued

*Received October 6, 1967. The results presented in this paper were obtained in the course of research sponsored under Contract No. N00014-67A-0109-0003, Task NR 064-496 by the Office of Naval Research, Washington, D. C. 
constant $m$-dimensional vectors; $\beta_{\gamma}$ are complex numbers; and $C_{i k}$ denote complex constant $m \times m$ matrices satisfying

$$
C_{0 n}=I, \quad \sum_{i=1}^{l}\left\|C_{i n}\right\| \leq \delta_{1}<1 .
$$

In (3) $I$ is the unit matrix and $\|C\|$ denotes the norm of the matrix $C$. If the elements of $C$ are $c_{p d}$, then $\|C\|$ is defined as

$$
\|C\|=\max _{p} \sum_{q=1}^{m}\left|c_{p q}\right| \text {. }
$$

The quantities $\alpha_{j}$ in (1) are complex numbers satisfying

$$
\alpha_{0} \equiv 0, \quad \operatorname{Re} \alpha_{i}>0 \quad(j=1,2, \cdots, l) .
$$

We note that Valeev [1] presented a method for obtaining asymptotic solutions of (1) satisfying certain initial conditions; general solutions of (1), however were not discussed. The emphasis in this paper is on the latter, which are important in the study of certain boundary value problems.

3. Main theorems. Consider the following definitions:

Definition 1. Let the matrices $L_{i}(s)$ and $A_{i}(s)$ be defined as

$$
\begin{aligned}
& L_{j}(s)=\sum_{k=0}^{n} s^{k} C_{j k} \quad(j=0,1,2, \cdots, l) \\
& A_{j}(s)=-L_{0}^{-1}(s) L_{j}\left(s+\alpha_{i}\right) \quad(j=1,2, \cdots, l)
\end{aligned}
$$

Definition 2. Let the complex numbers $\rho_{i}(i=1,2, \cdots, m n)$ be defined as the roots of the equation $\operatorname{det} L_{0}(s)=0$, i.e.

$$
\operatorname{det} L_{0}(s) \equiv \prod_{i=1}^{m n}\left(s-\rho_{i}\right)=0 .
$$

Further, let $\Lambda_{i}(i=1,2, \cdots, m n)$ represent the eigenvectors of

$$
L_{0}(s) \Lambda=0 .
$$

Here $\Lambda$ is a constant $m$-dimensional vector (the $\rho_{i}$ are of course the eigenvalues of (8)). We shall assume that each $\Lambda_{i}$ is normalized in such a way that one element is unity.

Definition 3. Let the matrices $L_{0}^{*}(s)$ and $R_{i}(i)$ be defined as

$$
\begin{aligned}
L_{0}^{-1}(s) & \left.=\frac{L_{0}^{*}(s)}{\prod_{i=1}^{m n}\left(s-\rho_{i}\right)} \text { (i.e., } L_{0}^{*}(s)=\text { adjoint of } L_{0}(s)\right) \\
R_{j}(i) & =-\frac{L_{0}^{*}\left(\rho_{i}\right) L_{j}\left(\rho_{i}+\alpha_{j}\right)}{\prod_{u=1 ; u \neq i}^{m n}\left(\rho_{i}-\rho_{u}\right)}(j=1,2, \cdots, l ; i=1,2, \cdots, m n)
\end{aligned}
$$

Definition 4. Let the vectors $P_{\gamma}^{*}(s)$ be defined as

$$
P_{\gamma}^{*}(s)=L_{0}^{-1}(s) P_{\gamma} \quad(\gamma=1,2, \cdots, \lambda) .
$$

Definition 5. Let the elements of any matrix $C_{i k}$ be denoted as $c_{p q}^{(j, k)}$ and let $R_{1}$ represent any compact set in the space of the parameters $c_{p q}^{(j, k)}, \alpha_{i}(p, q=1,2, \cdots, m$; 
$j=0,1, \cdots, l ; k=0,1, \cdots, n)$ where $\rho_{i}$ and $\left(\rho_{i}-\rho_{r}\right)^{-1}(i, r=1,2, \cdots, m n)$ are holomorphic functions of $c_{p q}^{(0, k)}$.

Definition 6. Let $R_{2}$ denote any compact set in the space of the parameters $c_{p e}^{(i, k)}, \alpha_{i}$ where $\rho_{i}$ and $\left(\rho_{i}-\rho_{r}-m_{1} \alpha_{1}-m_{2} \alpha_{2}-\cdots m_{l} \alpha_{l}\right)^{-1}\left(m_{t}=0,1,2, \cdots\right.$; $t=1,2, \cdots, l$; range of $p, q, j, k, i, r$ as in Definition 5) are holomorphic functions of $c_{p a}^{(0, k)}$ and $\alpha_{i}$.

Definimion 7. Let $R_{3}$ denote any compact set in the space of the parameters $c_{p a}^{(i, k)}, \alpha_{i}, \beta_{\gamma}, P_{\gamma \cdot}$ (the $v$ th component of the vector $P_{\gamma}$ ), where $\rho_{i}$ and $\left(\beta_{\gamma}-\rho_{i}-m_{1} \alpha_{1}-\right.$ $\left.m_{2} \alpha_{2}-\cdots-m_{l} \alpha_{l}\right)^{-1}\left(m_{t}=0,1,2, \cdots ; t=1,2, \cdots, l ; \gamma=1,2, \cdots, \lambda, v=1,2, \cdots, m\right.$; range of $p, q, j, k, i$ as in Definition 5) are holomorphic functions of $c_{p q}^{(0, k)}, \alpha_{i}, \beta_{\gamma}$.

Definition 8. Let $R_{4}$ denote any compact set in the space of the parameters $c_{p a}^{(j, k)}, \alpha_{i}, P_{\gamma}$ where $\rho_{i}$ and $\left(\beta_{\gamma}-\rho_{i}\right)^{-1}$ are holomorphic functions of $c_{p a}^{(0, k)}, \alpha_{i}, \beta_{\gamma}$ (range of $p, q, j, k, \gamma$ as in Definition 7.)

With the aid of Definitions 1-8 we assert

Theorem 1 (Complementary Solution). If $\left(c\left(\begin{array}{c}i k \\ p q\end{array}\right), \alpha_{i}\right) \in R_{2}$, then the general complementary solution of (1) can be written in the form

$$
Y(x)=\sum_{i=1}^{m n} \exp \left(\rho_{i} x\right) Z_{i}(x) h_{i}
$$

where $h_{i}$ are arbitrary scalar constants of integration and

$$
\begin{aligned}
& Z_{i}(x)=\left\{I+\sum_{i_{1}=1}^{l} A_{i_{1}}\left(\rho_{i}-\alpha_{i_{1}}\right) \exp \left(-\alpha_{i_{1}} x\right)\right. \\
& +\sum_{i_{1}, i_{1}=1}^{l} A_{i_{1}}\left(\rho_{i}-\alpha_{i_{1}}-\alpha_{i_{3}}\right) A_{i_{2}}\left(\rho_{i}-\alpha_{i_{3}}\right) \exp \left[-\left(\alpha_{i_{1}}+\alpha_{i_{3}}\right) x\right]
\end{aligned}
$$

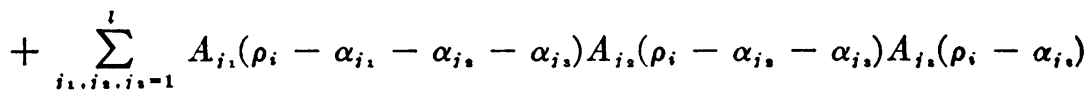

$$
\begin{aligned}
& \left.\cdot \exp \left[-\left(\alpha_{i_{1}}+\alpha_{i_{1}}+\alpha_{i_{3}}\right) x\right]+\cdots\right\} \Lambda_{i}
\end{aligned}
$$

which can be written as

$$
Z_{i}(x)=\left\{I+\sum_{N=1}^{\infty} \sum_{i_{1}, \ldots, j_{N}-1}^{l} \prod_{r=1}^{N}\left[A_{i r}\left(\rho_{i}-\sum_{i=r}^{N} \alpha_{i t}\right) \exp \left(-\alpha_{i r} x\right)\right]\right\} \Lambda_{i} .
$$

The series (12) has the following properties:

(i) It and the series resulting from a term by term differentiation of any order with respect to $x$, is absolutely and uniformly convergent ${ }^{1}$ with respect to both $\left(c_{p a}^{(j, k)}, \alpha_{i}\right)$ and $x$ in $x \geq 0$.

(ii) The sum, $Z_{i}(x)$, is $C^{\infty}$ in $x$ in $0 \leq x<\infty$ and holomorphic in $\left(c_{p q}^{(i, k)}, \alpha,\right)$.

(iii) It admits the following asymptotic representation as $x \rightarrow \infty$

$$
Z_{i}(x) \sim h_{i} \Lambda_{i}\{1+O[\exp (-\alpha x)]\}
$$

where $\alpha=\min _{i} \operatorname{Re} \alpha_{i}(j=1,2, \cdots, l)$.

${ }^{1} \mathrm{Here}$ and in the remaining text, all remarks concerning convergence shall apply to the series representing the components (elements) of the vector $Y(x)$. Unless otherwise stated, convergence is in the sense of Cauchy. 
(iv) The $N$ th partial sum, $S_{N}$, of the series of matrices within the brackets \{ \} of (12) can be obtained by use of the following recurrence relation

$$
\begin{aligned}
S_{1} & =I \\
S_{2}\left(\rho_{i}\right) & =I+\sum_{i=1}^{l} A_{j}\left(\rho_{i}-\alpha_{i}\right) \exp \left(-\alpha_{i} x\right), \\
S_{N}\left(\rho_{i}\right) & =I+\sum_{i=1}^{l}\left[S_{N-1}\left(\rho_{i}-\alpha_{i}\right) A_{i}\left(\rho_{i}-\alpha_{i}\right) \exp \left(-\alpha_{i} x\right)\right], \quad N>2 .
\end{aligned}
$$

In (14) we have indicated the functional dependence of $S_{v}$ on $\rho_{i}$ (suppressing the remaining parameters) by $S_{N}\left(\rho_{i}\right)$. The notation $S_{N}\left(\rho_{i}-\alpha_{i}\right)$ implies replacement of $\rho_{i}$ by $\rho_{i}-\alpha_{i}$.

Remark 1. A necessary condition that $\left(c_{p a}^{(i, k)}, \alpha_{i}\right) \in R_{2}$ is that the roots $\rho_{1}$ be distinct and no zeros of $\left(\rho_{i}-\rho_{r}-m_{1} \alpha_{1}-m_{2} \alpha_{2}-\cdots-m_{l} \alpha_{l}\right),(i, r=1,2, \cdots, m n$; $\left.m_{\imath}=0,1,2, \cdots ; t=1,2, \cdots, l\right)$ occur in the parameter space of interest.

REMARK 2. For computational purposes the recurrence relations (14) should be used to obtain the $N$ th partial sum of (12) if $l$ is large. Use of (14) can result in a reasonably efficient computational scheme; a term-by-term computation via (12), however, leads to an extremely cumbersome and inefficient process for large $l$.

Theorem 2 (Particular Solution). If $\left(c_{p q}^{(i, k)}, \alpha_{i}, \beta_{\gamma}, P_{\gamma_{\bullet}}\right) \in R_{3}$, then a particular solution of (1) is

$$
Y(x)=\sum_{\gamma=1}^{\lambda} \exp \left(\beta_{\gamma} x\right) V_{\gamma}(x)
$$

where

$$
V_{\gamma}(x)=\left\{\sum_{N=1}^{\infty} \sum_{i_{1}, \cdots, i_{N=1}} \prod_{r=1}^{N}\left[A_{i r}\left(\beta_{\gamma}-\sum_{i=r}^{N} \alpha_{i_{l}}\right) \exp \left(-\alpha_{i,} x\right)\right]\right\} P_{\gamma}^{*}\left(\beta_{\gamma}\right) .
$$

Equation (16), when expanded, has the form (12a) with $\rho_{i}$ replaced by $\beta_{\gamma}$ and $\Lambda_{i}$ by $P_{\gamma}^{*}\left(\beta_{\gamma}\right)$.

The series (16) has the following properties:

(i) It, and the series resulting from a term by differentiation of any order with respect to $x$, is absolutely and uniformly convergent with respect to both $\left(c_{p r}^{(i, k)}, \alpha_{i}\right.$, $\left.\beta_{\gamma}, P_{\gamma}\right)$ and $x$ in $x \geq 0$.

(ii) The sum, $V_{\gamma}(x)$, is $C^{\infty}$ in $x$ in $x \geq 0$ and holomorphic in $\left(c_{p a}^{(j, k)}, \alpha_{j}, \beta_{\gamma}, P_{\gamma_{0}}\right)$.

(iii) It admits the following asymptotic representation as $x \rightarrow \infty$

$$
V_{\gamma}(x) \sim P_{\gamma}^{*}\left(\beta_{\gamma}\right)\{1+O[\exp (-\alpha x)]\}
$$

where $\alpha=\min _{j} \operatorname{Re} \alpha_{i}(j=1,2, \cdots, l)$.

(iv) The $N$ th partial sum, $S_{N}$, of the series of matrices within the brackets \{ \} of (16) can be obtained by use of the recurrence relation

$$
\begin{aligned}
S_{1} & =I, \\
S_{2}\left(\beta_{\gamma}\right) & =I+\sum_{i=1}^{l} A_{i}\left(\beta_{\gamma}-\alpha_{j}\right) \exp \left(-\alpha_{j} x\right),
\end{aligned}
$$




$$
S_{N}\left(\beta_{\gamma}\right)=I+\sum_{i=1}^{l}\left[S_{N-1}\left(\beta_{\gamma}-\alpha_{j}\right)\right]\left[A_{i}\left(\beta_{\gamma}-\alpha_{i}\right) \exp \left(-\alpha_{j} x\right)\right], \quad N>2 .
$$

RemarK 3. A necessary condition that $\left(c_{p a}^{(i, k)}, \alpha_{j}, \beta_{\gamma}, P_{\gamma_{0}}\right) \in R_{3}$ is that no zeros of $\left(\beta_{\gamma}-\rho_{i}-m_{1} \alpha_{1}-m_{2} \alpha_{2}-\cdots-m_{l} \alpha_{l}\right),(i=1,2, \cdots, m n ; \gamma=1,2, \cdots, \lambda$; $\left.m_{t}=0,1,2, \cdots: t=1,2, \cdots, l\right)$, occur in the parameter space of interest.

Theorem 3 (Complementary Solution). If $C_{i n} \equiv 0(j=1,2, \cdots, l)$ and $\left(c_{p q}^{(i, k)}, \alpha_{i}\right)$ $\in R_{1}$, then the general complementary solution of (1) can be written as

$$
Y=\sum_{i=1}^{m n} Y_{i}(x) g_{i}
$$

where $g_{i}(i=1,2, \cdots, m n)$ are scalar constants of integration and where

$$
Y_{i}(x)=\sum_{N=1}^{\infty}\left[Y_{i}(x)\right]_{N}
$$

The $N$ th partial sum, $S_{N}$, of the series (19b) is obtained by use of the following recurrence relation

$$
S_{0}=0,
$$

$S_{N}(x)=\Lambda_{i} \exp \left(\rho_{i} x\right)+\sum_{j=1}^{l} \sum_{i=1}^{m n} R_{j}(t) \int_{0}^{x} S_{N-1}(\xi) \exp \left[\rho_{t}(x-\xi)-\alpha_{j} \xi\right] d \xi, \quad N>0$

We list a few properties of the series (19b):

(i) It, and the series resulting from a term by term differentiation of any order with respect to $x$, converges absolutely and uniformly with respect to both $\left(c_{p a}^{(i, k)}, \alpha_{i}\right)$ and $x$ in any finite subinterval of $x \geq 0$.

(ii) The sum $Y_{i}(t)$ is $C^{\infty}$ in $x$ and holcmorphic in $\left(c_{p a}^{(i, k)}, \alpha_{i}\right)$ in any finite subinterval of $x \geq 0$.

(iii) It admits the following asymptotic representation as $x \rightarrow \infty$

$$
Y_{i}(x) \sim \exp \left(\rho_{i} x\right)\left\{\sum_{r=1}^{m n} E(i, r) g_{r}[1+O(f(x) \exp (-\alpha x))]\right\}
$$

where $f(x)$ is a polynomial in $x$ of finite degree and $\alpha=\min _{i} \operatorname{Re} \alpha_{i}(j=1,2, \cdots, l)$. Here $E(i, r)$ is the vector

$$
\begin{aligned}
& E(i, r)=\left[I \delta_{i r}+\sum_{i_{1}=1}^{l} R_{i_{1}}(i) b_{i, r ; i_{1}}\right. \\
& +\sum_{j_{1}, j_{2},-1}^{l} \sum_{k_{2}=1}^{m n} R_{j_{1}}(i) R_{i_{3}}\left(k_{1}\right) b_{i, k_{2} ; j_{1}} b_{i, r ; j_{1}, j}
\end{aligned}
$$

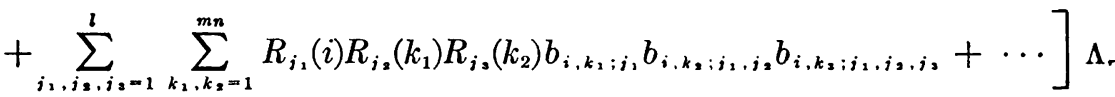

which can be written as

$$
\begin{aligned}
& E(i, r)=\left\{I \delta_{i, r}+\sum_{i_{2}=1}^{l} R_{j_{1}}(i) b_{i, r ; j_{2}}\right. \\
& +\sum_{N=2}^{\infty}\left[\sum_{i_{1}, \ldots, j_{N=1}}^{l} \sum_{k_{1}, \ldots, k_{N-1}=1}^{m n} R_{j_{2}}(i)\right. \\
& \left.\left.\times\left(\prod_{q=2}^{N} R_{j_{q}}\left(k_{a-1}\right)\right)\left(\prod_{t=1}^{N-1} b_{i, k_{t} ; i_{2}, \cdots, i_{l}}\right) b_{i, i_{i} i_{2}, \cdots, i_{N}}\right]\right\} \Lambda_{r}
\end{aligned}
$$


In Eqs. (22) $\delta_{i r}$ represents the Kronecker delta and the scalar constants $b_{i, r ; i_{1}, \cdots, i_{N}}$ are defined as

$$
\begin{aligned}
& b_{i, r, i_{1}, i_{2}, \cdots, i_{N}} \\
& =\left(\rho_{i}-\rho_{r}+\alpha_{i_{1}}+\alpha_{i_{2}}+\cdots+\alpha_{i_{N}}\right)^{-1} \\
& 0 \quad \text { if }\left(\rho_{i}-\rho_{r}+\alpha_{i_{1}}+\cdots+\alpha_{i_{N}}\right) \neq 0, \\
& =\quad \text { if }\left(\rho_{i}-\rho_{r}+\alpha_{i_{1}}+\cdots+\alpha_{i_{N}}\right)=0 .
\end{aligned}
$$

(iv) If $C_{j k}=0$ for $k \geq k^{*}(j=1,2, \cdots, l)$, then we have

$$
d^{t} Y_{i}(0) / d x^{t}=\rho_{i}^{t} \Lambda_{i} g_{i} \quad\left(t=0,1,2, \cdots, m\left(n-k^{*}\right)\right) .
$$

REMARK 4. A necessary condition that $c_{p q}^{(j, k)} \in R_{1}$ is that the roots $\rho_{i}$ be distinct.

REMARK 5. Since the integrands of (20) and (21) consist only of exponentials and products of exponentials and polynomials, the integrations can be carried out analytically.

REMARK 6. In most boundary value problems defined on the domain $0 \leq x<\infty$, it is necessary to satisfy a quiescent condition of the form $Y_{i}(x) \rightarrow 0$ as $x \rightarrow \infty$. From (21) it is clear that this condition can only be satisfied if the constant vector multiplying any exponential, $\exp \left(\rho_{i} x\right)$, with $\operatorname{Re} \rho_{i} \geq 0$ vanishes. This appears to lead to $m$ equations for $m n$ constants, $g_{r}$. This, however, is not the case. For a given $i$, the elements of the vector $\sum_{r=1}^{m n} E(i, r) g_{r}$ are linearly dependent, and only one equation for the $g_{r}$ need be satisfied. This equation can be selected as any one of the elements.

REMARK 7. Although the resultant form may not be as elementary as (24), the series for any $t$ th derivative of $Y_{i}(x)$ with respect to $x$ truncates at $x=0$.

Theorem 4 (Particular Solution). If $C_{i n} \equiv 0(j=1,2, \cdots, l)$ and $\left(c_{p q}^{(i, k)}\right.$, $\left.\boldsymbol{\alpha}_{j}, \beta_{\gamma}, P_{\gamma_{0}}\right) \in R_{4}$, then a particular solution of (1) is

$$
Y(x)=\sum_{N=1}^{\infty} Y_{N}(x)
$$

The $N$ th partial sum, $S_{N}$, of the series (25) is obtained from the following recurrence relation

$$
\begin{aligned}
S_{0} & \equiv 0 \\
S_{N}=\sum_{\gamma=1}^{\lambda} P_{\gamma}^{*}\left(\beta_{\gamma}\right) & \exp \left(\beta_{\gamma} x\right) \\
& +\sum_{j=1}^{l} \sum_{t=1}^{m n} R_{i}(t) \int_{0}^{x} S_{N-1}(\xi) \exp \left[\rho_{t}(x-\xi)-\alpha_{i} \xi\right] d \xi, \quad N>0 .
\end{aligned}
$$

The series (25) has the following properties:

(i) It, and the series resulting from a term by term differentiation of any order with respect to $x$, converge absolutely and uniformly with respect to both $\left(c_{p a}^{(i, k)}, \alpha_{i}\right.$, $\left.\beta_{\gamma}, P_{\gamma_{v}}\right)$ and $x$ in any finite subinterval of $x \geq 0$.

(ii) The sum, $Y(x)$, is $C^{\infty}$ in $x$ and holomorphic in $\left(c_{p q}^{(i, k)}, \alpha_{i}, \beta_{\gamma}, P_{\gamma}\right)$ in any finite subinterval of $x \geq 0$.

4. Proofs of the main theorems. 4.1. Laplace Transform. To facilitate the proofs of Theorems 1-4, we first reduce (1) to a system of linear difference equations by ap- 
plication of the Laplace transform. Defining the transform of the vector $Y(x)$ as

$$
\bar{Y}(s)=\int_{0}^{\infty} \exp (-s x) Y(x) d x, \quad \operatorname{Re} s>c=\text { const, } x>0
$$

one obtains, for the transformed version of (1),

$$
\sum_{i=0}^{\ell} L_{i}\left(s+\alpha_{i}\right) \bar{Y}\left(s+\alpha_{i}\right)=G(s)+H(s) .
$$

where

$$
\begin{aligned}
G(s) & =\sum_{\gamma=1}^{\lambda}\left[P_{\gamma} /\left(s-\beta_{\gamma}\right)\right], \\
H(s) & =\sum_{j=0}^{l} \sum_{r=0}^{n-1} \sum_{k=r+1}^{n}\left(s+\alpha_{j}\right)^{k-r-1} C_{j k} Y^{(r)}\left(0^{+}\right) . \\
L_{j}(s) & =\sum_{k=0}^{n} s^{k} C_{j k} \quad(j=0,1,2, \cdots, l) .
\end{aligned}
$$

In (28c), $Y^{(r)}\left(0^{+}\right)$is the $r$ th derivative of $Y(x)$ with respect to $x$, evaluated at $x=0$ as a limit from the right. In what follows, the quantities $Y^{(r)}\left(0^{+}\right)$are carried through the analysis as known constant vectors. They eventually emerge as constants of integration.

If (28a) is multiplied by $L_{0}^{-1}(s)$, one obtains the following system of difference equations for the transform, $\bar{Y}(s)$

$$
\bar{Y}(s)=\Phi(s)+\sum_{i=1}^{l} A_{j}(s) \bar{Y}\left(s+\alpha_{i}\right)
$$

where

$$
\begin{aligned}
A_{i}(s) & =-L_{0}^{-1}(s) L_{i}\left(s+\alpha_{i}\right) \quad(j=1,2, \cdots, l, \\
\Phi(s) & =L_{0}^{-1}(s)[G(s)+H(s)] .
\end{aligned}
$$

We now state and prove a lemma concerning the solutions of (29).

Lemma. The solution of (29) which is holomorphic in $\operatorname{Re} s>b(b=$ sufficiently large constant) and vanishes uniformly in $\operatorname{Im} s$ as $\operatorname{Re} s \rightarrow \infty$ in $\operatorname{Re} s>b$, is (1) unique, (2) can be obtained by the method of successive iterations, and (3) has the form

$$
\bar{Y}(s)=\Phi(s)+\sum_{N=1}^{\infty}\left\{\sum_{i_{1}, \cdots_{,}, i_{N=1}}^{l} \prod_{r=1}^{N}\left[A_{i_{r}}\left(s+\sum_{q=0}^{r-1} \alpha_{j_{a}}\right)\right] \Phi\left(s+\sum_{i=1}^{N} \alpha_{j_{t}}\right)\right\} .
$$

The series (31) is absolutely and uniformly (with respect to $s$ ) convergent when Re $s>b$.

REMARK 8. It is easily demonstrated that, since the solutions of (1) are of exponential order, the transform $\bar{Y}(s)$ must be holomorphic in Re $s>b(b=$ sufficiently large constant) and vanish uniformly in $\operatorname{Im} s$ as $\operatorname{Re} s \rightarrow \infty$ in $\operatorname{Re} s>b$.

Proof of Lemma. We verify the lemma as follows:

(i) Since the elements of $L_{j}(s)$ and $H(s)$ are polynomials in $s$, the only singularities in $A_{j}(s)$ and $\Phi(s)$ are isolated poles at the locations $s=\rho_{i}(i=1,2, \cdots, m n)$ and 
$s=\beta_{\gamma}(\gamma=1,2, \cdots, \lambda)$. Further $A_{i}(s)$ and $\Phi(s)$ are holomorphic and satisfy the following conditions uniformly in $\operatorname{Im} s$ when $\operatorname{Re} s>b$ :

$$
\begin{gathered}
\operatorname{Lim}_{\text {Re } s \rightarrow \infty} \Phi(s)=0, \\
\operatorname{Lim}_{\text {Res }} \sum_{i=1}^{l}\left\|A_{i}(s)\right\|<\delta_{2}<1 .
\end{gathered}
$$

Condition (32b) follows from (3).

(ii) Let $\Re$ represent the space of vector valued functions which are: (1) holomorphic for $\operatorname{Re} s>b$ and (2) vanish uniformly in $\operatorname{Im} s$ as $\operatorname{Re} s \rightarrow \infty$ in $\operatorname{Re} s>b$. Next, consider the operator $F[\bar{Y}(s)]$, defined by the right-hand side of (29); i.e. let

$$
F[\bar{Y}(s)]=\Phi(s)+\sum_{i=1}^{l} A_{i}(s) \bar{Y}\left(s+\alpha_{i}\right) \cdot
$$

In view of (i) above, and since $\operatorname{Re} \alpha_{i}>0$, it is evident that the operator $F$ maps an element of the space $\mathfrak{T}$ into an element of the same space.

(iii) Let the norm of any vector $\bar{Y}(s) \in \mathbb{M}$ be defined as

$$
\|\bar{Y}\|=\max _{k}\left|\bar{y}_{k}\right|
$$

where $\bar{y}_{k}(k=1,2, \cdots, m)$ are the elements of $\bar{Y}$. Next, let the metric between any two vectors $\bar{Y}_{1}(s), \bar{Y}_{2}(s) \in \Re$ be defined as

$$
\rho\left[\bar{Y}_{1}(s), \bar{Y}_{2}(s)\right]=\sup _{\diamond \in \operatorname{Re} s>b}\left\|\bar{Y}_{1}(s)-\bar{Y}_{2}(s)\right\| \cdot
$$

Use of the metric (35) implies any fundamental sequence in $\mathfrak{T}$ converges uniformly in the sense of Cauchy and therefore represents a point in $\mathfrak{T}$. The metric space $\mathfrak{T}$ is thus complete.

(iv) We estimate $\rho\left(F_{1}, F_{2}\right)$, where $F_{a}$ denotes $F\left[\bar{Y}_{q}(s)\right](q=1,2)$.

$$
\begin{aligned}
\rho\left(F_{1}, F_{2}\right)=\sup _{\varepsilon \in \operatorname{Re} s>b}\left\|\sum_{j=1}^{l} A_{i}(s)\left[\bar{Y}_{1}\left(s+\alpha_{j}\right)-\bar{Y}_{2}\left(s+\alpha_{j}\right)\right]\right\| \\
\leq\left(\sup _{a \in \operatorname{Re} s>b} \sum_{i=1}^{l}\left\|A_{j}(s)\right\|\right)\left(\sup _{s \in \operatorname{Re} s>b}\left\|\bar{Y}_{1}(s)-\bar{Y}_{2}(s)\right\|\right) \\
\quad=\left(\sup _{s \in \operatorname{Re} s>b} \sum_{i=1}^{l}\left\|A_{j}(s)\right\|\right) \rho\left(\bar{Y}_{1}(s), \bar{Y}_{2}(s)\right) .
\end{aligned}
$$

Combining this result with (32b) we conclude

$$
\rho\left(F_{1}, F_{2}\right) \leq \epsilon \rho\left(\bar{Y}_{1}, \bar{Y}_{2}\right),
$$

where $\epsilon<1$ for sufficiently large $b$.

Items (i) through (iv) imply the operator $F$, defined in a complete metric space $\mathfrak{T}$, maps the elements of $\mathfrak{T}$ into elements of the same space in such a way that (37) holds. Thus (29) is a contraction mapping for sufficiently large $b$. The Lemma follows by recourse to a well-known theorem due to $\mathrm{S}$. Banach on contraction mapping and fixed points [5, p. 27]. The fixed point of the operator $F[\bar{Y}(s)]$ is obtained by constructing a sequence of approximations, $\bar{S}_{i}$, according to

$$
\bar{S}_{0}(s)=0 ; \quad \bar{S}_{N}(s)=\Phi(s)+\sum_{i=1}^{l} A_{i}(s) \bar{S}_{N-1}\left(s+\alpha_{i}\right) .
$$


The absolute and uniform convergence of the sequence follows from the definition of the metric $\rho$.

4.2 Theorem 1. The form (11) and the series (12) are obtained from (31) by setting $G(s)=0$ [see (30) and (28b)] and by a term-by-term inversion of the resulting transform by residues. Following the inversion, the vectors $\Lambda_{i}$ are seen to be constant vectors depending on the initial data $Y^{(r)}\left(0^{+}\right), \rho_{i}$, and other parameters occuring in (1) (the form of $\Lambda_{i}$ is not pertinent to the discussion). Treating the $\Lambda_{i}$ as constant vectors of integration, and substituting the resulting series solutions for $Y(x)$ into the differential equations (1), one finds the differential equations are formally satisfied ${ }^{2}$ for each $i=$ $1,2, \cdots, m n$ if the $\Lambda_{i}$ are the eigenvectors of (8). Since the $\rho_{i}$ are assumed to be distinct, one concludes that $m n$ independent functions $Y_{i}(x)$ are obtained, and that (11) represents the general homogeneous solution of (1), contingent upon the proper convergence of (12). The latter point is discussed next.

Employing the norm (3) for matrices and the norm (34) for vectors, we obtain, from (12), the estimate

$$
\left\|Z_{i}(x)\right\| \leq\left\{1+\sum_{N=1}^{\infty} \sum_{i_{1}, \cdots, i_{N=1}}^{l} \prod_{r=1}^{N}\left[\left\|A_{i_{r}}\left(\rho_{i}-\sum_{i=r}^{N} \alpha_{i_{t}}\right)\right\| \mid \exp \left(-\alpha_{i, r} x\right)\right]\right\}\left\|\Lambda_{i}\right\| \cdot
$$

Consider the products occurring in each $N$ th term of (39). If $\left(c_{p q}^{(i, k)}, \alpha_{i}\right) \in R_{2}$, then, in view of (3) and (5), there exists an $N^{*}$ and $K\left(<N^{*}\right)$ depending only on the set $R_{2}$, such that for $N>N^{*}$ and $r=1$ to $N-K$ we have

$$
\sum_{j=1}^{l}\left\|A_{i}\left(\rho_{i}-\sum_{i=r}^{N} \alpha_{i t}\right)\right\|<\delta<1
$$

for all combinations of the $\alpha_{i_{t}}\left(j_{t}=1,2, \cdots, l\right)$ occurring in the argument of $A_{i}$. Noting that the form (40), for $r=N-K$ to $r=N$, is bounded by some constant $M^{*}$, and $\left\|\Lambda_{i}\right\|$ is bounded by some constant $M^{* *}$ if $\left(c_{p a}^{(j, k)}, \alpha_{j}\right) \in R_{2}$, one therefore obtains

$$
\left\|Z_{i}(x)\right\| \leq\left\|S_{N}^{*}\right\|+\left(M^{* *}\right)\left(M^{*}\right) \delta+\left(M^{* *}\right)\left(M^{*}\right) \delta^{2}+\left(M^{* *}\right)\left(M^{*}\right) \delta^{3}+\cdots
$$

Here $S_{N}^{*}$ is the sum of the first $N^{*}$ terms of (12).

The series (41) in a geometric series in $\delta$ which is convergent since $\delta<1$. Noting that each term of the component series of (12) is dominated by (41), we conclude that the component series of (12) are absolutely and uniformly convergent with respect to both $\left(c_{p a}^{(j, k)}, \alpha_{i}\right)$ and $x$ when $\left(c_{p q}^{(i, k)}, \alpha_{i}\right) \in R_{2}$ and $x \geq 0$. Further, since each term of (12) is holomorphic in $\left(c_{p q}^{(i, k)}, \alpha_{j}\right)$ and continuous in $x$ when $\left(c_{p q}^{(j, k)}, \alpha_{j}\right) \in R_{2}$, the uniform convergence of the series demonstrates that the sum has these same properties. Similar remarks apply to the series obtained from a term-by-term derivative of (12) of any order with respect to $x$. Thus, one concludes parts (i) and (ii) of Theorem 1. Part (iii) of Theorem 1 is obtained by observing the series (12) in the limit as $x \rightarrow \infty$. The recurrence relation (Part (iv)) is easily verified by successive substitution to obtain (12).

Remark 9 (Error Estimate). A similar procedure as used above yields the following estimate for the of the norm remainder, $\left\|R_{N}\right\| \equiv \| Z_{i}(x)-S_{N}^{*}(x) ! \mid$, of the series (12) after $N$ terms $\left(N>N^{*}\right)$ :

$$
\left\|R_{N}\right\| \leq M^{*} M^{* *} \delta^{N-N^{*}} /(1-\delta) \quad\left(\delta<1 \text { for } N>N^{*}\right) .
$$

${ }^{2}$ Set $A_{j}=\lambda A_{j}{ }^{*}$. Then (12) is a power series in $\lambda$. Substitution of the series for $Y(x)$ into (1) yields that (1) is formally satisfied for each order of $\lambda$. 
4.3. Theorem 2. To obtain the particular solution (15) and the series (16) we set $H(s)=0$ [see (30) and (28c)] and, again, invert (31) term by term by residues (a complementary solution, obtained in the process of the inversion, is omitted). The solution is verified by direct substitution into (1). Since the proof of convergence, etc., follows closely that for Theorem 1, the details will not be presented. An estimate for the remainder of the series (16) after $N$ term can be obtained by use of (42) with $\rho_{i}$ and $\Lambda_{i}$ replaced by $\beta_{\gamma}$ and $P_{i}^{*}\left(\beta_{\gamma}\right)$ respectively.

4.4. Theorem 3. Consider equation (38) with $G(s)=0$. If we (1) invert the first term of (38) by residues, (2) invert $A_{i}(s)$ by residues (this is a valid operation if $C_{\text {in }}=0$ ( $j=1,2, \cdots, l)$ since the elements of $A_{i}(s)$ are then the ratios of polynomials, the denominators being of higher order than the numerators) and then convolute the result with the inversion of $\bar{S}_{N-1}\left(s+\alpha_{i}\right)$ (which is $\exp \left(-\alpha_{j} x\right) S_{N}(x)$ if we denote as $S_{N}(x)$ the inversion of the partial sum $\bar{S}_{N}$ ) we immediately obtain the recurrence relation (20).

The series generated by (20) has the form

$$
\begin{aligned}
Y_{i}(x)= & W_{i}(x)+\int_{0}^{x} \kappa\left(x, \xi_{1}\right) W_{i}\left(\xi_{1}\right) d \xi_{1}+\int_{0}^{x} \kappa\left(x, \xi_{1}\right) \int_{0}^{\xi_{1}} \kappa\left(\xi_{1}, \xi_{2}\right) W_{i}\left(\xi_{2}\right) d \xi_{2} d \xi_{3} \\
& +\cdots+\int_{0}^{x} \kappa\left(x, \xi_{1}\right) \int_{0}^{\xi_{1}} \kappa\left(\xi_{1}, \xi_{2}\right) \cdots \int_{0}^{\xi_{N-1}} \kappa\left(\xi_{N-1}, \xi_{v}\right) W_{i}\left(\xi_{N}\right) d \xi_{N} \cdots d \xi_{1}+\cdots
\end{aligned}
$$

where

$$
\begin{aligned}
\kappa(x, \xi) & =\sum_{j=1}^{l} \sum_{i=1}^{m n} R_{j}(t) \exp \left[\rho_{t}(x-\xi)-\alpha_{j} \xi\right], \\
W_{i}(x) & =\exp \left(\rho_{i} x\right) \Lambda_{i} .
\end{aligned}
$$

By direct substitution of (43) into the differential equations (1) with zero right-hand side, one verifies that (1) is formally satisfied for each $i=1,2, \cdots, m n$, if, again, the $\Lambda_{i}$ are the eigenvectors of (8). Since the roots $\rho_{i}$ are assumed distinct, one concludes that $m n$ independent functions $Y_{i}(x)$ are obtained, and therefore, that (43) represents the general complementary solution of (1), contingent upon the proper convergence of (43).

If $\left(c_{p a}^{(i, k)}, \alpha_{j}\right) \in R_{1}$, then we have $\|\kappa(x, \xi)\| \leq M_{1}$ on the rectangle $0 \leq x \leq x_{0} .0 \leq$ $\xi \leq x_{0}$, and $\left\|W_{i}(x)\right\| \leq M_{2}$ on $0 \leq x \leq x_{0}$; Here $x_{0} . M M_{1}, M I_{2}$ are positive constants $\left(x_{0}\right.$ can be arbitrarily large). We therefore obtain the following estimate for $\left\|Y_{i}(x)\right\|$ from (43):

$$
\begin{aligned}
\left\|Y_{i}(x)\right\| \leq M_{2}+M_{2} M_{1} x_{0}+ & M_{2} M_{1}^{2} x_{0}^{2} / 2 ! \\
& +M_{2} M_{1}^{3} x_{0}^{3} / 3 !+\cdots+M_{2} M_{1}^{N} x_{0}^{N} / N !+\cdots .
\end{aligned}
$$

Since the series of positive constants (46) is convergent and each term of (46) dominates the corresponding term in the component series for $Y_{i}(x)$, we conclude that the component series of (43) are absolutely and uniformly convergent with respect to both $\left(c_{p a}^{(j, k)}, \alpha_{i}\right)$ and $x$ when $\left(c_{p a}^{(j, k)}, \alpha_{i}\right) \in R_{1}$ and $0 \leq x \leq x_{0}$. Since each term of the series is holomorphic in $\left(c_{p_{a}}^{(i, k)}, \alpha_{j}\right)$ and continuous in $x$, the uniform convergence indicates the sum $Y_{i}(x)$, has these same properties. A similar procedure as used above and applied to the series resulting from a term-by-term differentiation of (43) of any order with respect to $x$ indicates $Y_{i}(x) \in C^{\alpha}$ in $x$ on $0 \leq x \leq x_{0}$. Thus parts (i) and (ii) of Theorem 3 are concluded. 
The representation (21) for the asymptotic behavior of $Y(x)$ (see part (iii) of Theorem 3 ) is readily obtained by performing the integration indicated in (43) and taking the limit as $x \rightarrow \infty$.

The form (24) for $d^{t} Y_{i}(0) / d x^{t}$ (see part (iv) of Theorem 3 ) is obtained by differentiation of the series (43) for $Y(x)$ and by use of the relation

$$
\sum_{\nu=1}^{N}\left[\rho_{\nu}^{m} / \prod_{a=1 ; \nu \neq a}^{N}\left(\rho_{\nu}-\rho_{q}\right)\right]=0 \text { for } N>m+1 \text { and } \rho_{\nu} \text { distinct. }
$$

Equation (47) can be verified by expanding the leading term in partial fractions.

4.5. Theorem 4. Equation (26) is obtained from (38) by setting $H(s)=0$ and following the steps outlined for obtaining (20) in the Theorem 3 discussion. Since the proof of Theorem 4 parallels closely that for Theorem 3, the details will not be presented.

5. Examples. In this section we consider a few elementary problems to illustrate the foregoing Theorems.

Example 1. Let us construct the general solution of the equation

$$
\frac{d y}{d x}\left(1+\epsilon e^{-x}\right)+y=0
$$

on $x>0$ where $y$ is a scalar function. Here $l, m, n=1, C_{01}=1, C_{00}=1, C_{11}=\varepsilon$, all other $C_{j k}=0$. From equations (6-9) we obtain: $L_{0}(s)=s+1, L_{1}(s)=\varepsilon s, \rho_{1}=-1$, $\alpha_{1}=1, A_{1}=-1$. Since $\rho_{1}-\alpha_{1} \neq 0$, Theorem 1 is applicable if $|\varepsilon|<1$ and we obtain from (12)

$$
y=e^{-x}\left[1-\epsilon e^{-x}+\epsilon^{2} e^{-2 x}-\epsilon^{3} e^{-3 x}+\cdots\right] h,
$$

which when summed becomes

$$
y=h e^{-x} /\left(1+\epsilon e^{-x}\right) .
$$

In (49) $h$ is an arbitrary scalar constant.

EXAmpLe 2. Let us construct the general solution of

$$
d y / d x+\left(a-b e^{-\alpha x}\right) y=0
$$

on $x>0$, where $y$ is a scalar function and $a, b, \alpha$ are real, positive constants. Here $n, m, l=1 ; C_{01}=1, C_{00}=a, C_{10}=-b$, all other $C_{i k}=0, \alpha_{1}=\alpha$. From Eqs. (6-9) we obtain $L_{0}(s)=s+a, \rho_{1}=a, L_{0}^{*}(s)=1, L_{1}(s)=-b, R_{1}(1)=b$. Since $C_{i 1}=0$ $(j=0,1)$, Theorem 3 is applicable and we obtain from (20) or (43) by grouping terms with like powers of $b$

$$
y=e^{-a x}\left[1+\Theta+\Theta^{2} / 2 !+\Theta^{3} / 3 !+\cdots+\Theta^{N} / N !+\cdots\right] g
$$

where $\Theta=(b / \alpha)\left(1-e^{-\alpha x}\right)$ and $g$ is an arbitrary constant of integration. Upon summing the series (51), one finds the general solution of $(50)$ is

$$
y=g e^{-x} \exp \left[(b / a)\left(1-e^{-\alpha x}\right)\right] .
$$

EXAmple 3. Consider the following boundary value problem

$$
\begin{gathered}
d^{2} y / d x^{2}-p^{2}\left(1+a e^{-\alpha x}\right) y=0, \\
y(0)=1, \quad \operatorname{Lim}_{x \rightarrow \infty} y(x)=0,
\end{gathered}
$$


where $y$ is a scalar function and $p, \alpha, a$ are complex numbers satisfying $\operatorname{Re} p>0, \operatorname{Re} \alpha>0$. Here $l, m=1, n=2, C_{02}=1, C_{00}=-p^{2}, C_{10}=-p^{2} a$, all other $C_{i k}=0$. From Eqs. (6-9) we have $L_{0}(s)=s^{2}-p^{2}, \rho_{1}=-p, \rho_{2}=+p, A_{1}(s) \equiv A(s)=a p^{2} / s^{2}-p^{2}$. Applying Theorem 1 we obtain the general solution of (53a) in the form

$$
y=\sum_{i=1}^{2} h_{i} \exp \left(\rho_{i} x\right) Z_{i}(x)
$$

where

$$
\begin{aligned}
Z_{i}(x)= & 1+A\left(\rho_{i}-\alpha\right) \exp (-\alpha . x)+A\left(\rho_{i}-2 \alpha\right) A\left(\rho_{i}-\alpha\right) \exp (-2 \alpha x) \\
& +\cdots+A\left(\rho_{i}-N \alpha\right) A\left(\rho_{i}-(N-1) \alpha\right) \cdots A\left(\rho_{i}-\alpha\right) \exp (-N \alpha x)+\cdots .
\end{aligned}
$$

We now apply the boundary conditions (53b) to evaluate $h_{1}$ and $h_{2}$. Since $\operatorname{Re} p>0$, (54) indicates we must require $h_{2} \equiv 0$ to satisfy $y(\infty)=0$. The remaining constant, $h_{1}$, is evaluated by application of the $x=0$ boundary condition, and the desired solution is obtained as

$$
y=\exp (-p x) Z_{1}(x) / Z_{1}(0) .
$$

Theorem 1 indicates the series for $Z_{1}(x)$ is absolutely and uniformly convergent with respect to both $x$ and the parameters $p, a, \alpha$ on any compact set of the parameter space and in $x \geq 0$. Further $Z_{1}(x)$ is $C^{\infty}$ in $x$ and holomorphic in $p, a, \alpha$.

Example 4. Iet us solve the boundary value problem

$$
\begin{gathered}
\left(1+\epsilon e^{-x}\right) d^{2} y / d y^{2}-\left(a^{2}+b e^{-x}\right) y=c e^{-x} \\
y(0)=0, \quad \operatorname{Lim}_{x \rightarrow \infty} y(x)=0 .
\end{gathered}
$$

Here $y$ is a scalar function and $\epsilon, a, b, c$ are assumed to be real, positive constants. For this case we have $l, m, \lambda=1, n=2, C_{02}=1, C_{12}=\epsilon, C_{00}=-a^{2}, C_{10}=-b, P_{1}=c$, all other $C_{i k}$ and $P_{\gamma}=0 ; \alpha_{1}=\beta_{1}=1 ; L_{0}(s)=s^{2}-a^{2}, \rho_{1}=-a, \rho_{2}=+a, L_{1}(s)=$ $s^{2} \epsilon-b, A_{1}(s) \equiv A(s)=\left[b-\epsilon(s+1)^{2}\right] /\left(s^{2}-a^{2}\right), P^{*}(s)=c /\left(s^{2}-a^{2}\right)$. If $a \neq 1$ or 0 , then Theorems 1 and 2 can be used to obtain the complementary and particular solutions of (56a). For such cases the general solution of (56a) is readily obtained as

$$
y=\sum_{i=1}^{2} \exp \left(\rho_{i} x\right) Z_{i}(x) h_{i}+e^{-x} V(x)
$$

where $h_{i}$ represent constants of integration and

$$
\begin{aligned}
Z_{i}(x)= & 1+A\left(\rho_{i}-1\right) \exp (-x)+A\left(\rho_{i}-2\right) A\left(\rho_{i}-1\right) \exp (-2 x)+\cdots \\
& +A\left(\rho_{i}-N\right) A\left(\rho_{i}-(N-1)\right) \cdots A\left(\rho_{i}-1\right) \exp (-N x)+\cdots \\
V(x)= & \frac{c}{1-a^{2}}[1+A(0) \exp (-x)+A(-1) A(0) \exp (-2 x)+A(-2) A(-1) A(0) \\
& \cdot \exp (-3 x)+\cdots+A(-(N-1)) A(-(N-2)) \cdots A(0) \exp (-N x)+\cdots] .
\end{aligned}
$$

It is evident from (57) that we must require $h_{2} \equiv 0$ if $y(\infty)=0$ is to be satisfied. The remaining constant, $h_{1}$, is evaluated by application of the boundary condition 
$y(0)=0$. The resulting solution is

$$
y=-\left(V(0) / Z_{1}(0)\right) \exp (-a x) Z_{1}(x)+e^{-x} V(x) .
$$

The series (7) is convergent for $\epsilon<1$ and diverges if $\epsilon>1$.

6. Concluding remarks. The examples considered in Sec. 5 were of an elementary nature and were intended only to illustrate the various theorems presented. For examples concerning the application of Theorems 1-4 to the solution of several practical engineering problems, we refer the reader to [3], [4], [6] and [7]. In [3, 4], Theorem 3 was utilized in the investigation of the dynamic buckling of cylindrical shells (mathematically modeled by a nonlinear Donnell type shell theory) subjected to moving blast waves. In [6] Theorems 1 and 2 were employed in an investigation of the mechanical response (stress waves) of a thermoviscoelastic solid subjected to intense electromagnetic radiation. In [7] Theorem 1 was used in a study of one-dimensional wave propagation in an inhomogeneous elastic medium.

\section{REFERENCES}

1. K. G. Valeev, On linear differential equations with exponential coefficients and stationary delays of the argument. Regular case, PMM 26, No. 2, 1536-1553 (1962)

2. K. G. Valeev, On linear differential equations with exponential coefficients and stationary lags in the argument. Irregular case, PMM 26, No. 6, 1012-1024 (1962)

3. G. A. Hegemier, Instability of cylindrical shells subjected to axisymmetic moving loads, J. Appl. Mech. 33, 289-296 (1966)

4. G. A. Hegemier, Stability of cylindrical shells under moving loads by the direct method of Liapunov, J. of Appl. Mech., Paper No. 67-WA 1APM-3

5. L. Liusternik and V. Sobolev, Elements of functional analysis, New York, Frederick Ungar Publishing Co., 1961

6. G. A. Hegemier and L. W. Morland, Stress waves in a temperature dependent viscoelastic half-space subjected to impulsive electromagnetic radiation, Tech. Rpt. No. 4, AF-AFSOR-1226-67, Dept. of the Aerospace and Mechanical Engineering Sciences, Univ. of California, San Diego (to appear)

7. G. A. Hegemier and L. W. Morland, One-dimensional wave solutions for a nonhomogeneous semiinfinite medium, Tech. Rpt. No. 5, AF-AFOSR-1226-67, Dept. of the Aerospace and Mechanical Engineering Sciences, Univ. of California, San Diego, (to appear) 